Thomas Sühn*, Alfredo Illanes, Axel Boese, Christoph Lohmann, Ajay Pandey and Michael Friebe

\title{
Acoustic sensing of tissue-tool interactions - potential applications in arthroscopic surgery
}

\begin{abstract}
Arthroscopic surgery is a technically challenging but common minimally invasive procedure with a long learning curve and a high incidence of iatrogenic damage. These damages can occur due to the lack of feedback and supplementary information regarding tissue-instrumentcontact during surgery. Deliberately performed interactions can be used however to obtain clinically relevant information, e.g. when a surgeon uses the tactile feedback to assess the condition of articular cartilage. Yet, the perception of such events is highly subjective. We propose a novel proximally attached sensing concept applied to arthroscopic surgery to allow an objective characterization and utilization of interactions. It is based on acoustic emissions which originate from tissue-instrument-contact, that propagate naturally via the instrument shaft and that can be obtained by a transducer setup outside of the body. The setup was tested on its ability to differentiate various conditions of articular cartilage. A femoral head with varying grades of osteoarthritic cartilage was tapped multiple times ex-vivo with a conventional Veress needle with a sound transducer attached at the outpatient end. A wavelet-based processing of the obtained signals and subsequent analysis of distribution of spectral energy showed the potential of tool-tissue-interactions to characterize different cartilage conditions. The proposed concept needs further evaluation with a dedicated design of the palpation tool and should be tested in realistic arthroscopic scenarios.
\end{abstract}

Keywords: osteoarthritis, cartilage classification, surgical instrument, minimally invasive surgery, audio guidance.

https://doi.org/10.1515/cdbme-2020-3152

\footnotetext{
*Corresponding author: Thomas Sühn: Otto-von-Guericke University Magdeburg, Medical Faculty, Leipziger Str. 44, 39120 Magdeburg, Germany; E-Mail: thomas.suehn@ovgu.de Ajay Pandey: Queensland University of Technology (QUT), Brisbane, QLD, Australia. Michael Friebe, Alfredo Illanes, Axel Boese: Otto-von-Guericke University Magdeburg, Medical Faculty, Germany. Christoph Lohman: Department of Orthopaedic Surgery, University Hospital Magdeburg, Germany.
}

\section{Introduction}

Arthroscopic surgery is a well-established minimally invasive orthopaedic procedure for the diagnosis and treatment of human joints. In Germany, a total of 762,218 hospital admissions were recorded for arthroscopy in 2018 according to the health reporting system of the federal government [1], causing a high demand for skilled and experienced surgeons. However, especially for large joints such as the knee, the technically and ergonomically challenging nature of the procedure causes long learning curves.

A central procedural issue is the handling of surgical instruments, due to the restricted space within the joint capsule and the limited visibility in the arthroscopic field of view. While the navigation of instruments within the joint requires a substantial amount of experience, the surgeon simultaneously needs to manipulate the leg and arthroscope in order to access certain areas of the joint. While a range of surgical complications is reported, the majority of studies focus on postoperative problems. Especially intraoperative events such as iatrogenic cartilage damage due to instrument handling are difficult to assess. A survey with 93 participants unveiled high incidence rates perceived by the surgeons, with about $50 \%$ stating that iatrogenic damage occurs in 1 out of 10 cases [2]. Intraoperative feedback regarding cartilage-tool-interactions, could be of great importance for tool guidance and for mitigating detrimental effects.

While arthroscopy is not generally recommended for the management of knee osteoarthritis (OA) [3], an objective and minimally invasive assessment of the cartilage would come along with clinical benefits. Especially for post-traumatic OA, which is initiated by joint trauma e.g. cartilage, meniscus, and ligament tears and typically requires arthroscopic intervention. Currently, the grading of osteoarthritic cartilage is based on radiographic images (Kellgren-Lawrence score) or magnetic resonance imaging [4]. However, there likely is a discrepancy between subjective complaints of the patient and the diagnostic finding in those images, potentially underestimating cartilage damages [5]. More reliable classifications, such as the OARSI score [6], are based on the 
histopathological analysis of cartilage samples and thus can only be conducted postoperatively.

The most commonly used method for arthroscopic cartilage assessment is the Outerbridge classification, which is limited by the subjective visual nature of the technique [7]. Further, surgeons typically utilize the tactile feedback as additional assessment tool. While roughness and softening of the cartilage are important for appropriate grading, the perception of both is subjective and thus grading depends on the observer. In consequence, a poor inter- and intraobserver agreement remains the main limitation of this method [7]. However, incorporation of a simple technique for the objective assessment of those properties could enhance the reliability.

A novel, audio-based approach for the acquisition of information about tissue-instrument-interactions in minimally invasive surgery has been proposed by our research group [8]. The concept utilizes acoustic emissions (AE) which originate from the interaction between tissue and distal tip of any medical instrument, for example the tip of a needle during transcutaneous interventions. The emerging acoustic signals propagate naturally along the shaft of the interventional instrument and can be obtained via an audio transducer attached at the proximal end. Since the transducer remains outside of the body and in distance to the surgical side, the concept can be characterized as out-patient and passive acoustic sensing. The capability of the concept has been proven for various applications such as laparoscopic access [9], cardiac catheterization [10] or surface texture differentiation [11]. The aim of this work is to highlight the potential of this approach to overcome some of the mentioned limitations of arthroscopic surgery and cartilage classification.

\section{Materials and Methods}

Preliminary experiments were conducted to assess the feasibility of the introduced audio-based approach for the palpation and characterization of different articular cartilage conditions. A first prototype of a palpation instrument was built and evaluated in ex-vivo measurements on a human femoral head. The acquired audio signals were analysed to estimate their information content and capability to differentiate between various grades of OA.

\subsection{Transducer Setup}

A conventional Veress insufflation needle (KLS Martin $\mathrm{GmbH}$, Freiburg, Germany) with a length of $120 \mathrm{~mm}$ and a diameter of $2.0 \mathrm{~mm}$ was used due to its mechanical features and availability. The Veress needle (VN) is a spring-loaded needle widely used for laparoscopic access and consisting of two parts depicted in Figure 1 (A). The outer sharp cannula is connected spring-loaded to a hollow obturator $(D=1.6 \mathrm{~mm})$ gliding within the lumen of the cannula. This obturator has a blunt tip to protect tissue from injury by the sharp outer needle. Pressure exerted on the tip allows to overcome the spring force and pushes the obturator into the shaft of the cannula. This spring-loaded mechanism enables mechanical excitations due to interactions at the tip to propagate to some extent freely to the opposite end. As a side effect, the residual force caused by the resting spring was used to limit the force applied to palpate the cartilage tissue. The force restrains the obturator from moving and ranges around $\mathrm{F}=1 N[12]$.

The naturally propagated acoustic signal was obtained using a transducer setup containing a 3D-printed housing and a custom-built printed circuit board (PCB) for the transducer as shown in Figure 1 (B). The PCB incorporates a bottom-port electret-type MEMS microphone (SPH0645LM4HB Knowles Electronics, LLC, Illinois, US) with internal gain amplifier and 18-bit sigma-delta analog-digital-converter, providing a digital output via $\mathrm{I}^{2} \mathrm{~S}$ (inter integrated circuit sound) codec. The audio signal was acquired with a sampling frequency of fs $=44.1 \mathrm{kHz}$ and stored in WAV audio-format. The manufactured prototype is shown in Figure 1 (C).

The outer cannula was used to grasp the VN. As a result, effects and noise due to the touching of the instrument were reduced to a minimum. The setup ensured a closed cavity and defined volume for the propagation of the sound wave to the

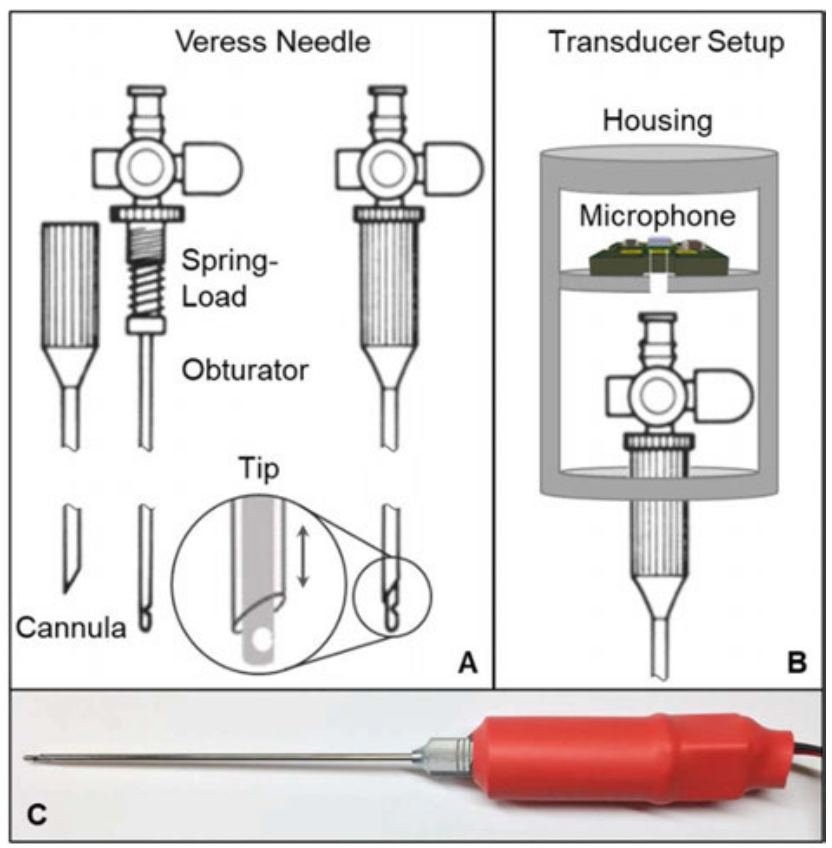

Figure 1 Mechanical features of a VN (A), transducer setup for the palpation instrument (B) and instrument prototype (C). 
air-coupled microphone. Further, the housing prevents environmental noise from affecting the obtained signal.

\subsection{Experimental Protocol}

A recently explanted human femoral head with different grades of OA was used for ex-vivo measurements with the palpation instrument. The cartilage conditions depicted in Figure 2 varied from preserved cartilage (a), mild OA (b), severe OA (c) to plain bone (d).

Each condition was palpated five consecutive times within the marked area. While the location was not exactly identical, similar duration, contact angle and force (less than $1 \mathrm{~N}$ ) were ensured manually. Despite the fact that the manual execution limits the reproducibility of the touching events, it corresponds with a realistic clinical scenario of the potential application.

\subsection{Signal Processing}

For pre-processing and removal of signal trending, a discrete wavelet transformation (DWT) with Daubechies mother wavelet of order 4 was used for decomposition. Afterwards, the signal was reconstructed excluding the scales which contained the very low frequent components. For the analysis, a continuous wavelet transformation (CWT) using a Morlet wavelet was performed. The scales corresponding to the pseudo-frequencies around $4 \mathrm{kHz}$ were utilized and the envelope was computed to detect the events of touching. Figure 3 depicts exemplarily the DWT of the detrended signal

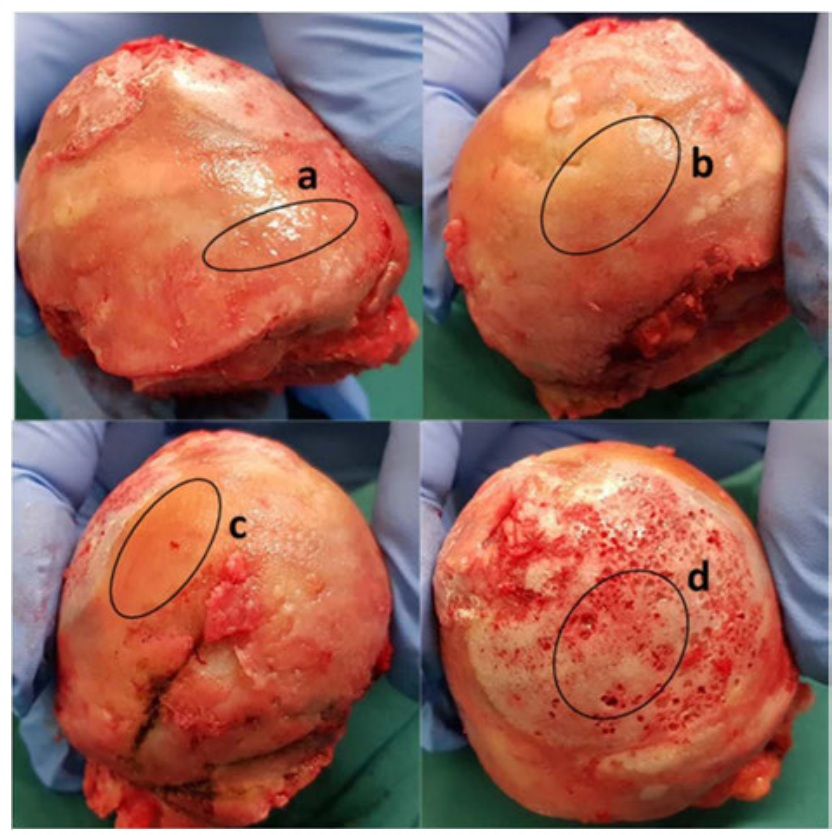

Figure 2 Femoral head with various grades of OA (a-d)



Figure 3 Pre-processing steps: CWT (top) and envelope for selected scales for event detection (bottom), cartilage c.

and the computed envelop for cartilage condition c. At the specific time of each event a windowing of $300 \mathrm{~ms}$ was applied to the pre-processed signal. Subsequently, a CWT using Morlet wavelet was computed to inspect the spectral characteristic. A windowed signal and the computed CWT are shown in figure 4 . The spectrum was used to verify, if the respective cartilage condition generates a sort of signature of the energy contribution in different frequency bands. Hence, the signals were separated into four bands corresponding roughly to pseudo-frequencies of low LF: $15-70 \mathrm{~Hz}$, middle-low MLF: $70-400 \mathrm{~Hz}$, middle-high MHF: $400-$ $1600 \mathrm{~Hz}$, and high HF: $1600-7000 \mathrm{~Hz}$ frequencies. For each band the energy and the contribution to the total spectral energy of the event was calculated via the equation $E_{i} / E_{T}$ with $i=L F, M L F, M H F, H F$ and $E_{T}=\sum_{i} E_{i}$.

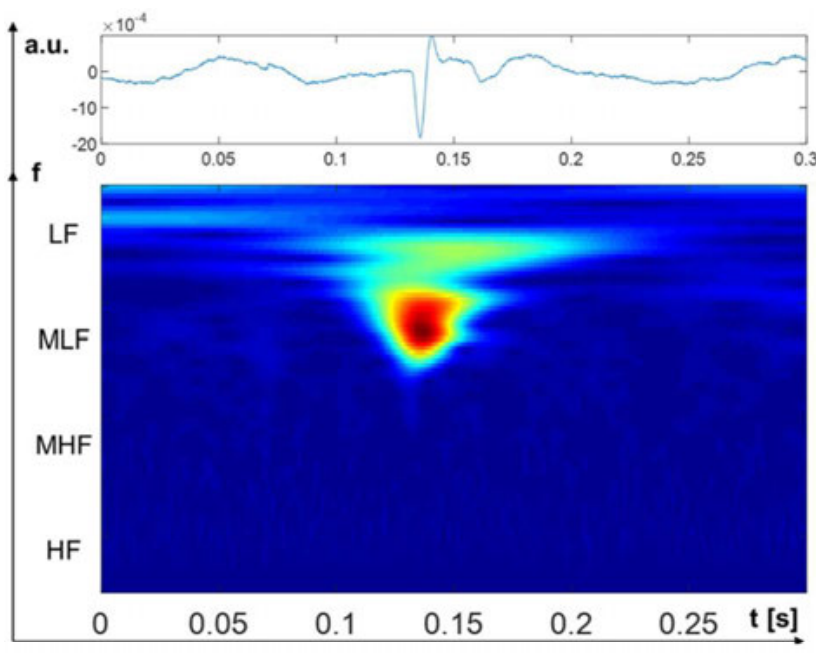

Figure 4 Windowed signal of a touching-event (top) and DWT for analysis of the distribution of spectral energy (bottom), cartilage $c$. 


\section{Results and Conclusion}

As shown in Figure 5, different types of events, e.g. touching of various grades of osteoarthritic cartilage, showed to produce different patterns in the time/scale spectrum resulting in different distributions of spectral energy during the excitation. This visible pattern reflects likewise in the contribution of the four separated bands LF to HF to the total spectral energy, depicted in Figure 6. While the energy trace appears to depend on the physical properties of the palpated cartilage, the pattern of each condition seems to be independent from the exact location of contact within the respective area. The setup and measurement approach need further evaluation with respect to the influence of parameters such as contact duration, -force and -angle, surface texture and inter- and intra-user variability. In summary, the presented audio-based method and transducer setup has shown its capability to detect instrument-tissue interactions in a simulated arthroscopic scenario. The proposed processing strategy provides promising preliminary results for an arthroscopic characterization of osteoarthritic cartilage.

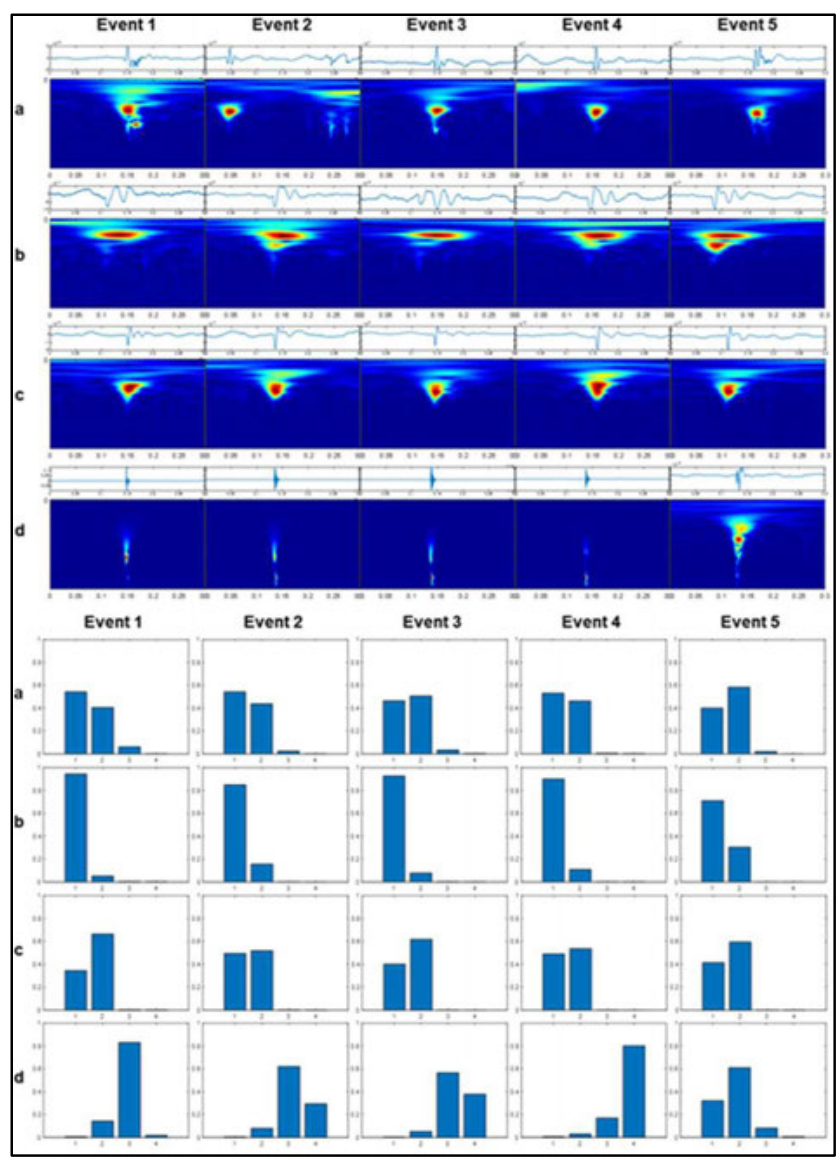

Figure 5 Top: Time-domain signals and time/scale spectra for five palpation-events of OA cartilage a-d. Bottom: Contribution of the separated bands (from left: LF to HF) to the total spectral energy.

\section{Author Statement}

Research funding: The author state no funding involved. The authors state no conflict of interest. The research related to human use complies with all relevant national regulations, institutional policies, was performed in accordance with the tenets of the Helsinki Declaration and has been approved by the authors' institutional review board.

\section{References}

[1] Statistisches Bundesamt. Gesundheitsberichterstattung des Bundes: Operationen und Prozeduren in Krankenhäusern. Entgeltsysteme im Krankenhaus, DRG-Statistik und PEPPStatistik 2019. Available at: www.gbe-bund.de Accessed March 11, 2020.

[2] Jaiprakash A, O'Callaghan WB, Whitehouse SL, Pandey A, Wu L, Roberts J, Crawford RW. Orthopaedic surgeon attitudes towards current limitations and the potential for robotic and technological innovation in arthroscopic surgery. J Orthop Surg (Hong Kong) 2017;25(1):2309499016684993.

[3] Katz JN, Brownlee SA, Jones MH. The role of arthroscopy in the management of knee osteoarthritis. Best Pract Res Clin Rheumatol 2014;28(1):143-156.

[4] Roemer FW, Eckstein F, Hayashi D, Guermazi A. The role of imaging in osteoarthritis. Best Pract Res Clin Rheumatol 2014;28(1):31-60.

[5] Abdelaziz H, Balde OM, Citak M, Gehrke T, Magan A, Haasper C. Kellgren-Lawrence scoring system underestimates cartilage damage when indicating TKA: preoperative radiograph versus intraoperative photograph. Arch Orthop Trauma Surg 2019;139(9):1287-1292.

[6] Pritzker KP, Gay S, Jimenez SA, Ostergaard K, Pelletier J-P, Revell PA, Salter D, van den Berg WB. Osteoarthritis cartilage histopathology: grading and staging. Osteoarthr Cartil 2006;14(1):13-29.

[7] Slattery C, Kweon CY. Classifications in Brief: Outerbridge Classification of Chondral Lesions. Clin Orthop Relat Res 2018;476(10):2101-2104.

[8] Illanes A, Boese A, Maldonado I, Pashazadeh A, Schaufler A, Navab N, Friebe M. Novel clinical device tracking and tissue event characterization using proximally placed audio signal acquisition and processing. Sci Rep 2018;8(1):12070.

[9] Schaufler A, Sühn T, Esmaeili N, Boese A, Wex C, Croner R, Friebe M, Illanes A. Automatic differentiation between Veress needle events in laparoscopic access using proximally attached audio signal characterization 2019;5(1):369-371.

[10] Mahmoodian N, Schaufler A, Pashazadeh A, Boese A, Friebe $M$, Illanes $A$. Proximal detection of guide wire perforation using feature extraction from bispectral audio signal analysis combined with machine learning. Comput Biol Med 2019;107:10-17.

[11] Chen $\mathrm{CH}$, Sühn T, Kalmar M, Maldonado I, Wex C, Croner $\mathrm{R}$, Boese A, Friebe M, Illanes A. Texture differentiation using audio signal analysis with robotic interventional instruments. Comput Biol Med 2019;112:103370.

[12] Schramel JP, Kindslehner A, Bockstahler BA, Dupré GP. Comparison of design features and mechanical properties of commercially available Veress needles. Vet Surg 2017;46(7):994-1001. 